\title{
Chemistry for nanotechnology
}

\author{
Grzegorz Schroeder*, Joanna Kurczewska \\ Adam Mickiewicz University in Poznań, Faculty of Chemistry, Umultowska 89b, 61-614 Poznań, Poland \\ "Corresponding author: e-mail: schroede@amu.edu.pl
}

\begin{abstract}
In recent years, the processing order during the synthesis of new chemical compounds has been redefined. Until now a chemist considered primarily receiving a new compound and only then searched for its potential application. The new philosophy of proceedings forces chemists to answer the question: what physical and chemical properties a new chemical compound must have, and what should be structured. After that it has to be planned how to get the compound including the defined budget. The compounds obtained by conventional chemical synthesis are then used to create new functional materials having the properties as scheduled. The paper presents the way of the proceedings from a molecular receptor to a new nanomaterial containing this receptor, so in other words from individual molecules to new material with specific and previously planned properties.
\end{abstract}

Keywords: nanotechnology, materials chemistry, supramolecular chemistry.

\section{INTRODUCTION}

Chemical Abstract Service (CAS) Registry ${ }^{1}$ in a division of the American Chemical Society is the gold standard for chemical substance information. In the real time every day CAS Registry on the American Chemistry Science servers displays the number of recorded chemical compounds obtained by chemists in the world. CAS Registry at July 2013 contains more than 730000000 unique organic and inorganic chemical substances, such as alloys, coordination compounds, minerals, mixtures, polymers and salts. CASREACT ${ }^{\circledR}$ system, produced by CAS, contains: more than 51.9 million single- and multi-step reactions - available with SciFinder and on STN, more than 13.6 million additional synthetic preparations available with SciFinder, CHEMLIST ${ }^{\circledR}$ (Regulated Chemicals Listing), produced by CAS, - an electronic collection of thousands of chemical substances that are regulated in key markets across the globe. CHEMLIST contains more than 297000 substances.

This huge number of chemicals obtained every day shows that a synthesis of chemical compound is not limited by the capabilities of chemists but it may be restricted only by budget. One can now ask the question: why the chemists continually strive to synthesize new chemical compounds, when 70 million chemical compounds are already obtained and the vast majority of them without determined properties.

At this point it creates a dilemma because chemists are interested in a compound itself, while customers of chemistry are interested in the chemical properties of a product and its influence on the environmental protection. The traditional methods of chemicals' synthesis satisfy the demand for products that are functional in large molecular systems. On the other hand, a dynamic development of nanotechnology enforces the necessity of receiving products with specific properties. In recent years, there has been redefined a processing order of working of a chemist during the synthesis of new chemical compounds. Until now receiving of a new compound has taken a priority over searching for a potential application.

The new philosophy of proceedings forces chemists to answer the question: what physical and chemical properties a new chemical compound should have and what should be structured, and then answer the question: how one can get it taking a budget under consideration. As opposed to the conventional chemical synthesis, a presentation of the applicability of molecular receptors during preparation of new functional materials promotes a new approach to the work of chemists. The basic research in which we define the properties of individual molecules and molecular receptors can become the beginning of an application of these compounds in the material chemistry. Additionally, it can lead to the synthesis of new materials with specific properties or a selective construction of the measuring nanosystems $\mathrm{s}^{2-4}$.

The process from the molecule, that is characterized by the well-studied properties, to modern material chemistry is limited only by the imagination of chemists and the demand for new organic materials in the nanotechnology as well as for a new generation of the selective measurement nanosystems.

Gaining knowledge about the synthesis methods of chemical compounds with planned construction site, both in terms of arrangement of atoms and functional groups, as well as spatial structure, allowed to obtain a number of new molecular receptor systems that are capable of creating host-guest complexes. The paper presents the way of the proceedings from a molecular receptor to a new nanomaterial containing this receptor, so in other words from individual molecules to a new material with specific and previously planned properties.

\section{RESULTS AND DISCUSSION}

Molecular receptors are systems capable of binding other molecules/ions (analytes) in active components of their structure forming host-guest complexes. The ability of analytes (guests) of binding to receptors depends on the degree of structure matching the host and guest molecules in terms of spatial and energy. The interaction energy of molecules in a host-guest complex is a measure of molecular recognition selectivity and a complex stability. Molecular receptors can be divided into several types according to the structure and the method of the guest molecule binding:

Receptors binding guest (analyte) molecules by covalent bonds in a reversible process, with a low equilibrium constant (Fig. 1); 

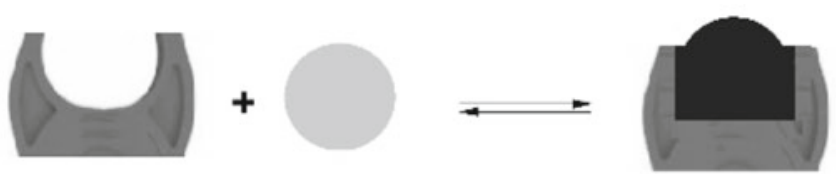

Figure 1. Schematic representation of the reversible covalent bond formation

Receptors binding guest molecules by non-covalent bonds:

- Acceptor receptors, having lone electron pair in their structure, forming donor-acceptor bonds with donor guest (analyte) molecules;

- Donor receptors, having a substituent in their structure capable of attachment of lone electron pair, forming donor-acceptor bonds with guest (analyte) molecules that carrying lone electron pair;

- Receptors having non-polar substituents forming weak hydrophobic bonds with non-polar guest (analyte) molecules;

- Receptors forming hydrogen bond with guest (analyte) molecules;

- Ionic receptors forming ionic bonds with cations, anions and salts;

- Receptors forming bonds with guest (analyte) molecules by spatial matching of reactants in host-guest complex.

The structures of receptors described and the type of interactions with an analyte are presented in Figure 2.
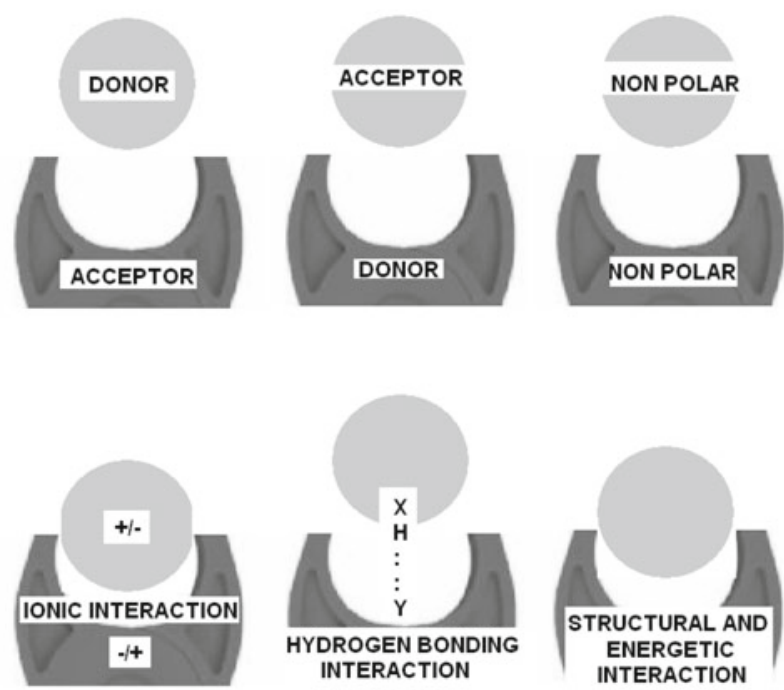

Figure 2. Different types of supramolecular interactions in host-guest complexes

Owing to an unlimited number of molecular receptors capable of selective binding of analytes with a different structure and properties, it is possible to apply them for the preparation of new nanomaterials. The receptors would be located surface on the of an inorganic carrier characterized by nanometric dimension. Such hybrid systems combine the physical properties of nonomaterials (dependant on the nanoparticle size) and the chemical properties of molecular receptors deposited on the surface. The properties of hybrid systems depend on the ratio of the surface atoms to the total number of atoms in the nanomaterial. All surface atoms of a nanomaterial can be used for the deposition of receptor molecules, thus the number of those atoms determines inorganic-organic properties of hybrid materials. A synthesis of hybrid systems requires the presence of reactive groups in a molecular receptor and an inorganic carrier capable of covalent bond formation while maintaining the structure and properties of that receptor. In order to maintain those properties and also the lability of organic part in solution, the receptor is often deposited on the surface indirectly through the linker (Fig. 3).

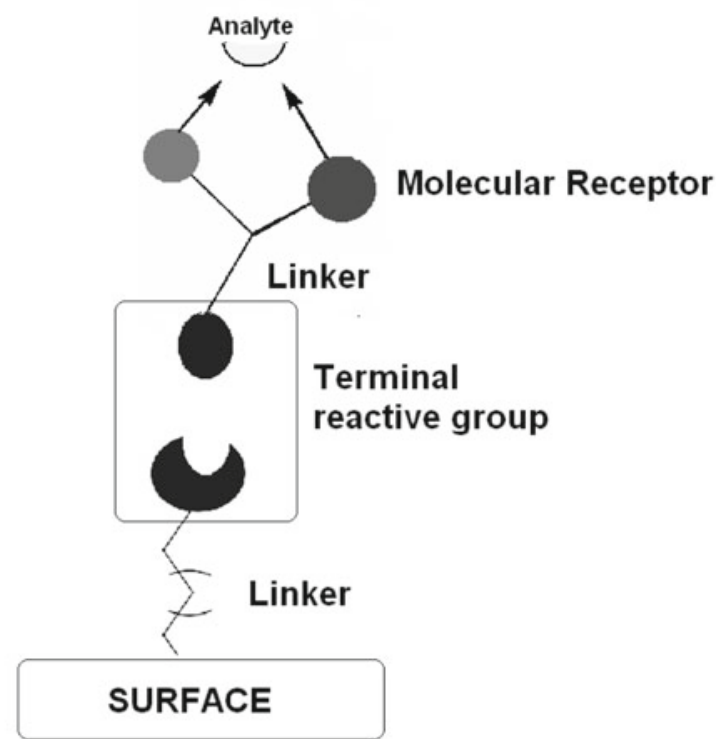

Figure 3. Schematic representation of a method of hybrid system formation

It is worth discussing wider the subject of surface functionalization by different types of linkers (Fig. 4) . $^{5}$ The first group, zero-length cross-linkers, are molecules responsible for the activation of surfaces. Within this group, surface activation by silanization with different reactive silanes is particularly popular. Hombifunctional cross-linkers have reactive groups at both sides in order to conjugate the same functional groups. They are used in excess in order to avoid blocking both reactive groups. On the other hand, heterobifunctinal cross-linkers are supposed to connect molecules with different functionalities. The last group consists of cross-linkers with three equal or different reactive groups for connecting of three chemical species.

Inorganic-organic hybrid materials with selective molecular receptors are obtained on the basis of silica, carbon nanomaterials, organosilicon polymers, magnetic particles, bio-organic polymers (chitosan, alginates), metals, semiconductors and quartz. Such numerous variety of nano-carriers as well as the possibility of surface functionalization by organic molecules that determine the chemical properties of new materials, set out the way to obtain materials with the desired properties. A clarification of nanomaterial properties determines a selection of an organic molecule used for functionalization. Visualization of the surface functionalization and the binding of analytes is shown in Figure 5.

The process from a molecule to a new nanomaterial with specified properties is carried out in several steps:

1. Synthesis of molecular receptor of specified chemical properties; 

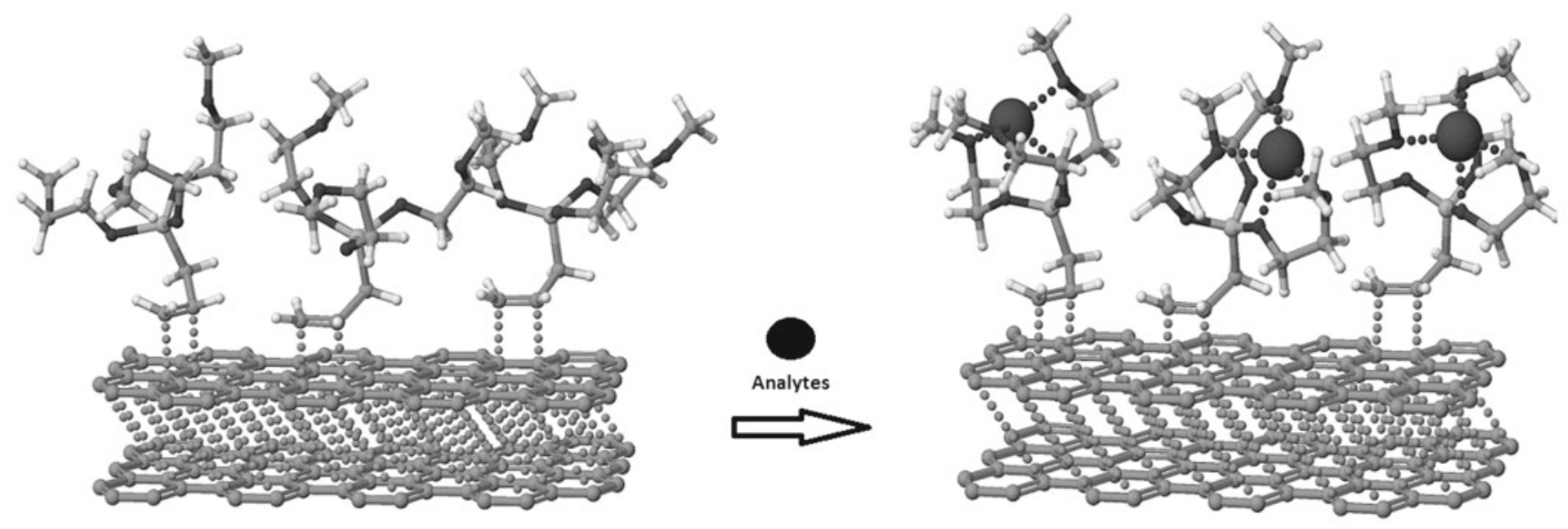

Ligands on the surface

Complexes on the surface

Figure 4. Examples of different types of linkers: A - zero-length; B - homobifunctional; C - heterobifunctional; D - trifunctional cross-linkers

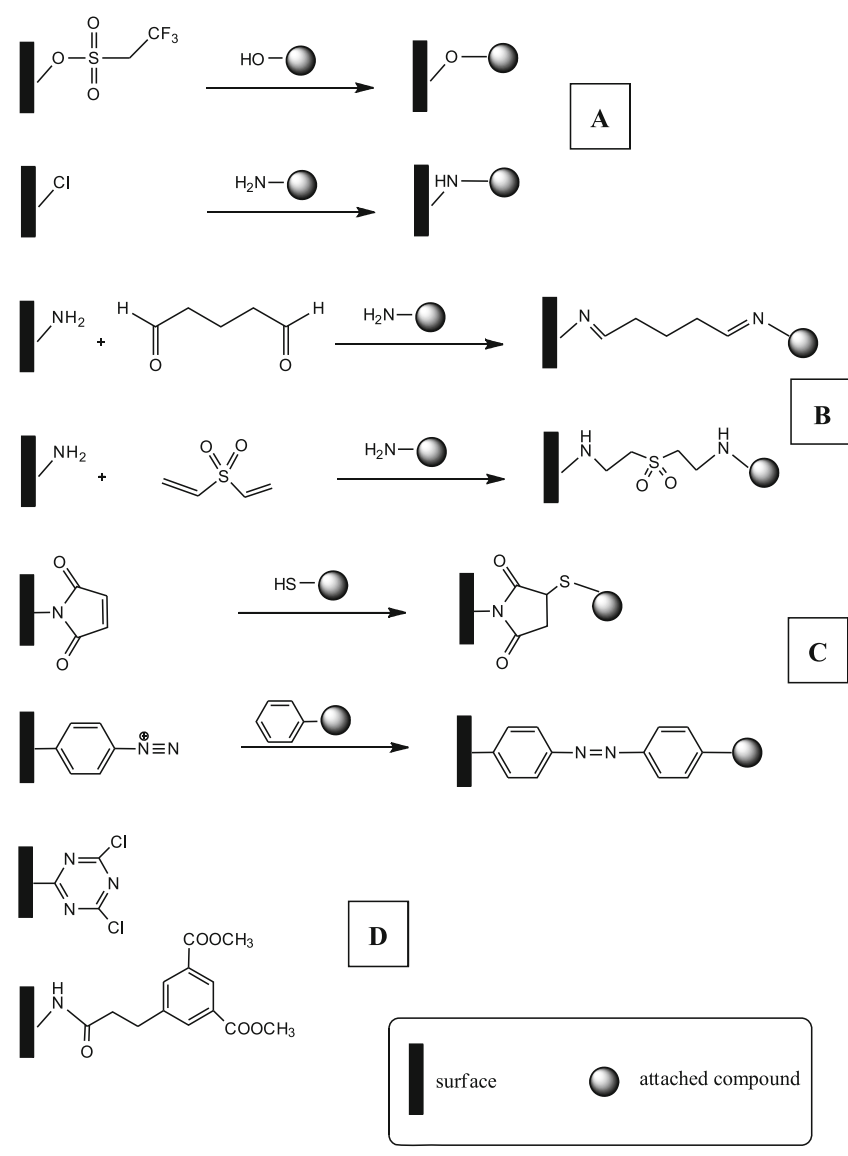

Figure 5. The functionalization of surface by ligand formed complexes with analytes

2. Modification of the receptor by an introduction to its structure a linker with a reactive terminal group;

3. Selection of nano-carrier according to the properties and the size of nanoparticles;

4. Functionalization or modification of nano-carrier in order to obtain reactive functional groups on the surface;

5. Synthesis of nanomaterial during substitution reaction of a receptor to a carrier surface;

6. Confirmation of assumed properties in practical applications.

The process can be described with a few examples.
Synthesis of nanomaterials capable of sugar bonding

Arylboronic acids ${ }^{\mathbf{6}-14}$ belong to a group of molecular receptors capable of sugar bonding in a reversible process with a covalent bond formation (Figure 6).

While the process of nanomaterials formation capable of sugar bonding is presented in Figure 7.

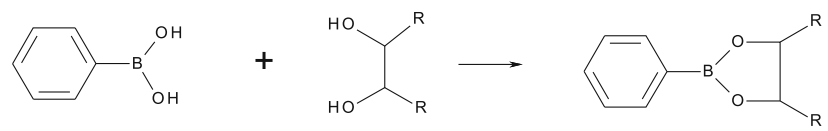

Figure 6. Schematic representation of sugar bonding by arylboronic acids
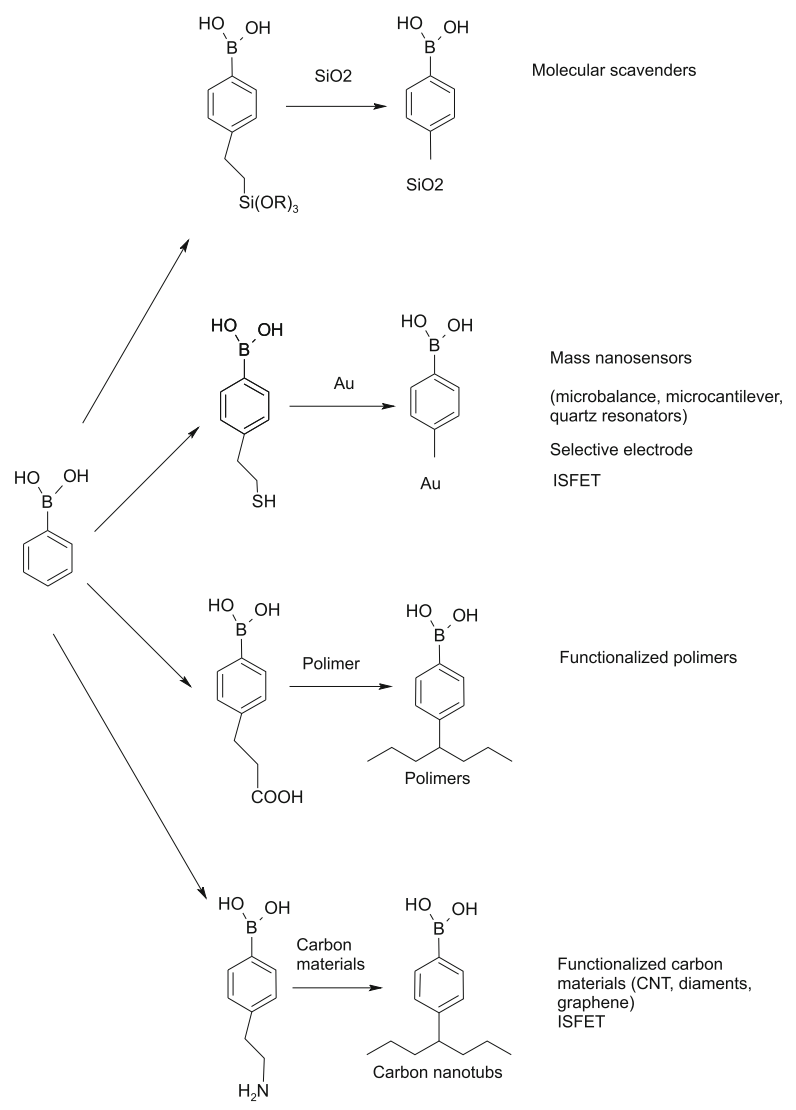

Figure 7. Schematic representation of a synthesis of nanomaterials used for sugar bonding 
Synthesis of nanomaterials capable of ion bonding, based on ionic bond

Derivatives of 2,2',6,2'-terpiridine ${ }^{15-22}$ belong to a group of molecular receptors capable of ion bonding in a reversible process with ionic bond formation (Fig. 8).<smiles>c1ccc(-c2cccc(-c3ccccn3)n2)nc1</smiles>

$$
\text { A } \mathrm{Fe}^{2+}
$$

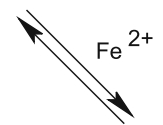

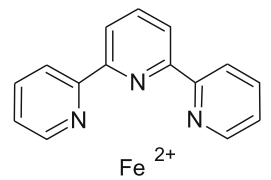

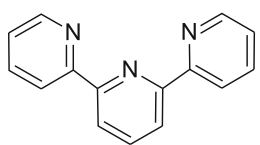

Figure 8. Reversible process of ionic bond formation

Molecules of a receptor can be deposited on the surface physically or chemically through covalent or ionic bonds (Fig. 9).

The process of a synthesis of 2,2',6,2'-terpiridine derivatives that are capable of bonding with surface is presented in Figure 10.

The exemplary nanomaterials described in this paper, capable of sugar or ion binding retain the properties of molecular receptors and nanocarriers.

A basic research in which we define the properties of individual molecules - molecular receptors - could initiate the use of these compounds in the synthesis of new materials with specific properties or designing of selective miniature measuring systems. The search by consumers of new materials with specified properties forces chemists to change their approach to a synthesis of chemicals. Currently, there is a demand for materials with specified properties rather than the synthesis of new materials with potential use. The way from a single

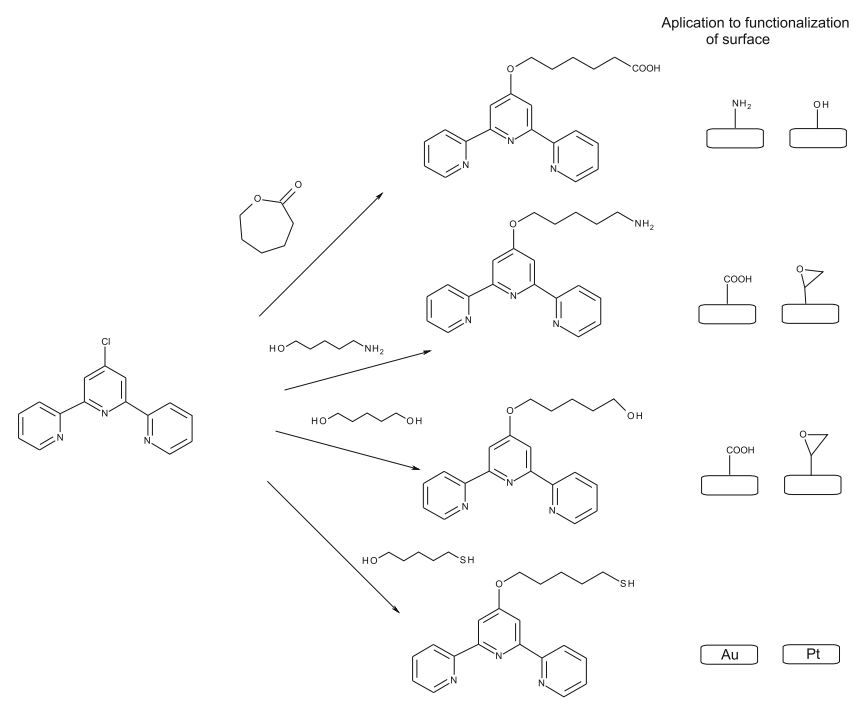

Figure 10. Schematic representation of 2,2',6,2'-terpiridine derivatives' synthesis applied for surface functionalization

molecule with the well-studied properties to nanotechnology depends only on the imagination of chemists and demands of consumers for new materials in chemical analysis, pharmacy, medicine and industry.

\section{ACKNOWLEDGEMENTS}

The authors would like to thank The National Science Center of Poland (Grant No. 2011/03/B/ST5/01573) for financial support.

\section{LITERATURE CITED}

1. The American Chemical Society; http://www.cas.org/

2. Schroeder, G. (2011). Molecular receptors. From receptor molecules to functional materials, Wiad. Chem. 65, 1021.

3. Molecular Receptors properties and applications. (2009). Ed. G. Schroeder, Cursiva, 2009, ISBN 978-83-62108-02-2.

4. Chemical functionalization of surfaces for nanotechnology (2011) Ed. G. Schroeder, Cursiva, ISBN 978-83-62108-07-7.

5. Heise, Ch. \& Bier, F.F. (2006). Immobilization of DNA on Microarrays, Top. Curr. Chem., 261, 1, DOI: 10.1007/128_007.

6. Takahashi, S. \& Anzai, J. (2005). Phenylboronic Acid Monolayer-Modified Electrodes Sensitive to Sugars, Langmuir 21, 5102, DOI: 10.1021/la050171n.

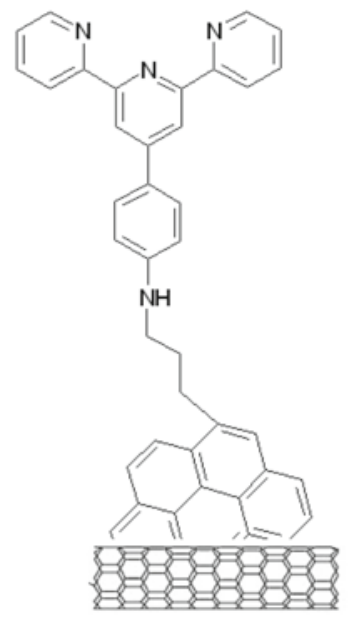

a)

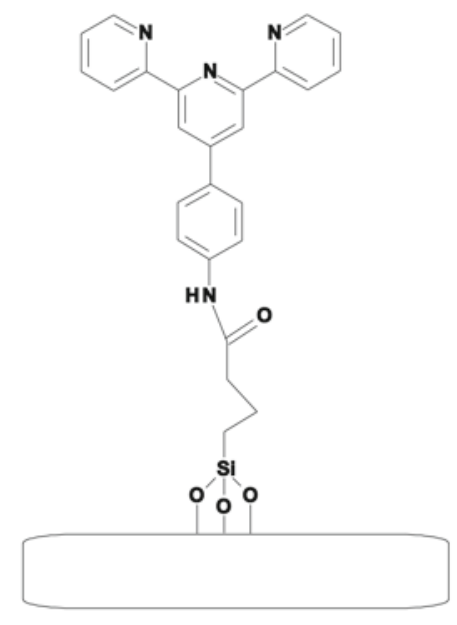

b)

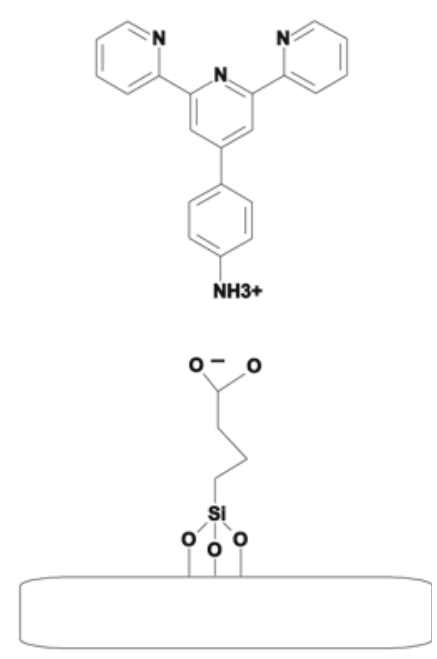

c)

Figure 9. Different types of bonds between 2,2',6,2'-terpiridine derivatives and a surface: (a) physical bonding with carbon material; (b) covalent bond with silicon/quartz surface; (c) ionic bond 
7. Barriet, D., Yam, C.M., Shmakova, O.E., Jamison, A.C. \& Lee, T.R. (2007). 4-Mercaptophenylboronic Acid SAMs on Gold: Comparison with SAMs Derived from Thiophenol, 4-Mercaptophenol, and 4-Mercaptobenzoic Acid, Langmuir 23, 8866, DOI: $10.1021 / 1 a 7007733$.

8. Baker, G.A., Desikan, R. \& Thundat, T. (2008). Label-free sugar detection using phenylboronic acid-functionalized piezoresistive microcantilevers, Anal. Chem. 80, 4860, DOI: 10.1021/ ac702588b.

9. Takahashi, S. \& Anzai, J. (2005). Phenylboronic acid monolayer-modified electrodes sensitive to sugars. Langmuir, 24, 5102, DOI: 10.1021/la050171n.

10. Boronic Acids. Preparation and Applications in Organic Synthesis and Medicine. Ed. D.G. Hall, WILEY-VCH Verlag GmbH \& Co. KGaA, Weinheim, 2005, ISBN-13:978-3-52730991-7.

11. Adamczak-Woźniak, A. (2010). Synthesis application and stability of phenylboronic esters (pp. 59-85), In Functionalized molecules - synthesis, proporties and application, Ed. V.I. Rybachenko, Schidnyj Wydawnyczyj Dim, Donetsk, ISSN 978-966-317-076

12. Adamczak-Woźniak, A. (2010). Phenylboronic compounds as molecular recognition and self-assembing agents (pp. 9-25), in Functionalized molecules - synthesis, proporties and application, Ed. V.I. Rybachenko, Schidnyj Wydawnyczyj Dim, Donetsk, ISSN 978-966-317-076.

13. Spożyński, A., Adamczak-Woźniak, A. \& Żubrowska, A. (2008). Interamolecular interactions in orto-aminomethylphenylboronic acids - potent sacharide receptor, (pp.75-91), In From concept to molecular receptor, Ed. V.I. Rybachenko, Schidnyj Wydawnyczyj Dim, Donetsk, ISSN 978-966-317-022-0.

14. Spożyński, A., Żubrowska, A. \& Adamczak-Woźniak, A. (2007). Synthesis of boronic acids-molecular receptor for sugars (pp. 51-89), In Synthetic receptors in molecullar recognition, Ed. V.I. Rybachenko, Schidnyj Wydawnyczyj Dim, Donetsk, ISSN 978-966-317-013-8.

15. Schubert, U.S., Hofmeier, H., Newkome, G.R. (2006). Modern Terpyridine Chemistry, Wiley-VCH Verlag GmbH \& Co.KGaA, Weinheim, ISBN-13:978-3-527-31475-1.

16. Constable, E.C. (2007). 2,2':6',2"-Terpyridines: From chemical obscurity to common supramolecular motifs, Chem. Soc. Rev., 36, 246, DOI: 10.1039/b601166g.

17. Harris, E.K. (2010). Polymer or Macrocycle? Cobalt Complexes of Ditopic 2,2':6',2" - Terpyridine Ligands with Flexible Spacers, $\mathrm{PhD}$ thesis, University of Basel, Basel.

18. Chiper, M. (2008). Advanced supramolecular assemblies based on terpyridine metal complexes: Understanding reaction parameters and designing new materials, $\mathrm{PhD}$ thesis, Technische Universiteit Eindhoven,

19. Hofmeier, H. \& Schubert, U.S. (2004). Recent developments in the supramolecular chemistry of terpyridine-metal complexes, Chem. Soc. Rev., 30, 373, DOI: 10.1039/b400653b.

20. Schubert, U.S. \& Eschbaumer, C. (2002). Macromolecules containing bipyridine and terpyridine metal complexes: towards metallosupramolecular polymers, Angew. Chem. Int. Ed., 41, 2892, DOI: 10.1002/1521-3773.

21. Shunmugam, R., Gabriel, G.J., Aamer, K.A., Tew, G.N. (2010). Metal-ligand-containing polymers: terpyridine as the supramolecular unit, Macromol Rapid Commun., 12, 784, DOI: $10.1002 /$ marc.200900869.

22. Sakamoto, R., Katagiri, S., Maeda, H. \& Nishihara, H. (2013). Bis(terpyridine) metal complex wires: Excellent long-range electron transferability and controllable intrawire redox conduction on silicon electrode, Coord. Chem. Rev., 257, 1493, DOI: org/10.1016/j.ccr.2012.08.025. 